\title{
Entropy Production in Flames
}

\author{
VEDAT S. ARPACI and AHMET SELAMET
}

Department of Mechanical Engineering and Applied Mechanics, University of Michigan, Ann Arbor, MI 48109

Thermodynamic foundations of the thermal entropy production are rested on the concept of lost heat, $(Q / T) \delta T$. The thermomechanical entropy production is shown to be in terms of the lost heat and the lost work as

$\delta S_{\mathrm{g}}=\frac{1}{T}\left[\left(\frac{Q}{T}\right) \delta T+\delta W_{\mathrm{L}}\right]$,

where the second term in brackets denotes the lost (dissipated) work into heat.

The dimensionless number $\Pi_{s}$ describing the local entropy production $s$ in a quenched flame is found to be

$$
\Pi_{s} \sim\left(\mathrm{Pe}_{\mathrm{D}}^{0}\right)^{-2} \text {, }
$$

where $\Pi_{s}=s^{m} l^{2} / k, l=\alpha / S_{\mathrm{u}}{ }^{\circ}$ (a characteristic length), $k$ thermal conductivity, $\alpha$ thermal diffusivity, $S_{\mathrm{u}}{ }^{0}$ the adiabatic laminar flame speed at the unburned gas temperature, $\mathrm{Pe}_{\mathrm{D}}{ }^{0}=S_{\mathrm{v}}{ }^{0} \mathrm{D} / \alpha$ the flame Peclet number, and $D$ the quench distance.

The tangency condition $\partial \mathrm{Pe}_{\mathrm{b}}{ }^{0} / \partial \theta_{\mathrm{b}}=0$, where $\theta_{\mathrm{b}}=T_{\mathrm{b}} / T_{\mathrm{b}}{ }^{0}, T_{\mathrm{b}}$ and $T_{\mathrm{b}}{ }^{0}$ denoting, respectively, the burned gas (nonadiabatic) and adiabatic flame temperatures, is related to an extremum in entropy production. The distribution of entropy production between the flame and burner is shown in terms of the burned gas temperature and the distance from the burner.

\section{NOMENCLATURE}

$c_{\mathrm{p}} \quad$ specific heat at constant pressure

$d$ thickness of reaction zone

$D$ quench distance

$E$ activation energy

$f_{i}$ body force

$H$ enthalpy

$k$ thermal conductivity

$K \quad$ kinetic energy

$l$ a characteristic length

$p$ pressure

Pe Peclet number

$q_{i}$ heat flux in $x_{i}$

$Q$ heat flow

$R \quad$ universal gas constant

$s$ entropy/mass

$s^{m} \quad$ rate of entropy generation/volume

$s_{i j} \quad$ rate of deformation
$S \quad$ entropy

$S_{u} \quad$ laminar flame speed at unburned gas temperature

$t$ time

$T$ temperature

$u \quad$ internal energy/mass

$u^{\prime \prime \prime} \quad$ rate of energy gei eration/volume

$U$ internal energy

$v \quad$ specific volume

$v_{i} \quad$ velocity in $x_{i}$

$V$ volume

$W$ work

$x, x_{i}$ coordinate axis

\section{Greek Symbols}

$\begin{array}{ll}\alpha & \text { thermal diffusivity } \\ \theta & =T / T_{\mathrm{b}}^{0}\end{array}$

Copyright (C) 1988 by The Combustion Institute Published by Elsevier Science Publishing Co., Inc. 52 Vanderbilt Avenue, New York, NY 10017 
$\xi \quad$ variable defined by Eq. (37)

$\Pi$ entropy number

$\rho$ density

$\tau_{i j} \quad$ stress

$\Phi$ potential energy

\section{Subscripts}
A available
b burned
D quench distance
$\mathrm{g}, \mathrm{G}$ generation
L lost
s entropy
u unburned
I first order Ferguson and Keck model
II second order Ferguson and Keck model
III Clarke model

\section{Superseripts \\ K conduction \\ 0 adiabatic or stagnation}

\section{INTRODUCTION}

The foundations of entropy production go back to Clausius and Kelvin's studies on the irreversible aspects of the Second Law of Thermodynamics. Since then the theories based on these foundations have been elaborated by the efforts of natural philosophers and later utilized by applied scientists and engineers (see, for example, Bejan [1, 2] for applications involving heat transfer and fluid mechanics, and Arpaci [3, 4] and Arpaci and Selamet [5] for extension to gas radiation). Yet, the entropy production associated with flames appears to remain untreated and is the motivation of this study. As is well known, the entropy production results from dissipative processes (involving mass, species, momentum and/or heat transfer, electromagnetic, or nuclear transport). Less known is the fact that the dissipation may have a diffusive or hysteretic origin, the diffusion being directional and the hysteresis being cyclic. However, except for a few cases (such as strain hardening and magnetic saturation), the majority of dissipative processes including the one in flames is of diffusive nature and is the concern of the study.

The study consists of six sections: following this introduction, Section 2 clarifies the thermodynamic foundations of the entropy production, Section 3 develops the transport aspects of this production, Section 4 applies the entropy production to flame quenching and interprets the tangency condition of laminar flame quenching by an extremum in entropy production, Section 5 deals with the distribution of entropy production in quenched laminar flames, and Section 6 concludes the study.

\section{THERMODYNAMIC FOUNDATIONS}

Under the influence of thermomechanical effects only, the First Law of Thermodynamics for a differential control volume (Fig. 1a) is

$d H^{0}=\delta Q-\delta W$,

where

$H^{0}=U+p V+K+\Phi$

is the stagnation enthalpy, $V$ the volume, $K$ the kinetic energy and $\Phi$ the potential energy. The rest of the notation is conventional. For later convenience, rearrange the heat through control surface as

$\delta Q \equiv \delta\left[T\left(\frac{Q}{T}\right)\right]=T \delta\left(\frac{Q}{T}\right)+\left(\frac{Q}{T}\right) \delta T$

or as

$\delta Q=\delta Q_{\mathrm{A}}+\delta Q_{\mathrm{L}}$.

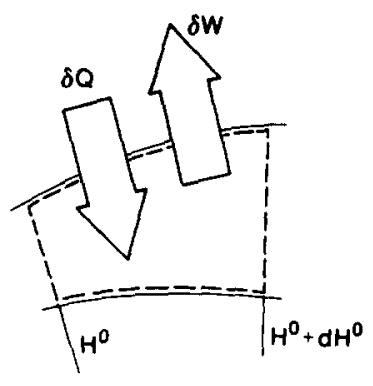

(a)

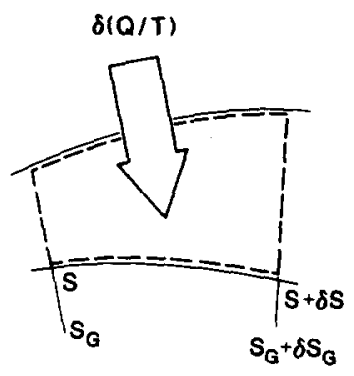

(b)
Fig. 1. First and Second Laws of Thermodynamics. 
Let

$\delta Q_{\mathrm{A}}=T \delta\left(\frac{Q}{T}\right) \quad$ and $\quad \delta Q_{\mathrm{L}}=\left(\frac{Q}{T}\right) \delta T$

denote the available ${ }^{1}$ (balanced) heat and the lost (dissipated) heat, respectively. Also, let

$\delta W=-\delta\left(W_{\mathrm{A}}+W_{\mathrm{L}}\right)$,

$\delta W_{\mathrm{A}}$ and $\delta W_{\mathrm{L}}$ being the available (balanced) work and the lost (dissipated) work related to stress (or shaft) (Fig. 2a). The explicit (tensorial) form of $\delta W$ (and other work terms) need to be delayed to the next section. Now, rearrange Eq. (1) in terms of Eqs. (4) and (6). Thus

$$
\begin{aligned}
d(U+K+\Phi)= & \left(\delta Q_{\mathrm{A}}+\delta Q_{\mathrm{L}}\right) \\
& -d(p V)+\left(\delta W_{\mathrm{A}}+\delta W_{\mathrm{L}}\right) .
\end{aligned}
$$

The mechanical energy balance, obtained either by eliminating thermal effects from Eq. (7) or directly from Newton's Second Law of Motion, is

$d(K+\Phi)=-V d p+\delta W_{\mathrm{A}}$.

Clearly, for a steady, incompressible and inviscid flow, Eq. (8) reduces to the Bernoulli equation.

For any (reversible or irreversible) process, the Second Law of Thermodynamics for the differential control volume (Fig. 1b) is

$d S=\delta\left(\frac{Q}{T}\right)+\delta S_{\mathrm{G}}$

1 No relation to the concept of availability. $\delta S_{\mathrm{G}}$ denoting the entropy production (or generation). For a reversible process, $\delta S_{\mathrm{G}}=0$ and $T=$ constant, and Eq. (9) reduces to the familiar form of the Second Law. Equation (9) multiplied with temperature,

$T d S=T \delta\left(\frac{Q}{T}\right)+T \delta S_{\mathrm{G}}$,

may be rearranged in terms of Eq. (5) to give the energy equivalent of the Second Law (Fig. 2b),

$T d S=\delta Q_{\mathrm{A}}+T \delta S_{\mathrm{G}}$.

Now, subtract Eqs. (8) and (11) from Eq. (7). Thus,

$d U-T d S=\delta Q_{\mathrm{L}}-p d V-T \delta S_{\mathrm{G}}+\delta W_{\mathrm{L}}$,

which, in view of the Gibbs (thermodynamic) relation,

$d U=T d S-p d V$

reduces to

$\delta S_{\mathrm{G}}=\frac{1}{T}\left(\delta Q_{\mathrm{L}}+\delta W_{\mathrm{L}}\right)$

This result shows the contribution of thermal dissipation, as well as that of mechanical dissipation, to entropy production [the sign of $\delta W_{\mathrm{L}}$ in Eq. (6) is assumed to yield the sign of $\delta W_{\mathrm{L}}$ in Eq. (14)]. Clearly, under the influence of chemical, electromagnetic, and nuclear effects, Eq. (14) needs to be augmented by including the dissipation resulting from these effects.

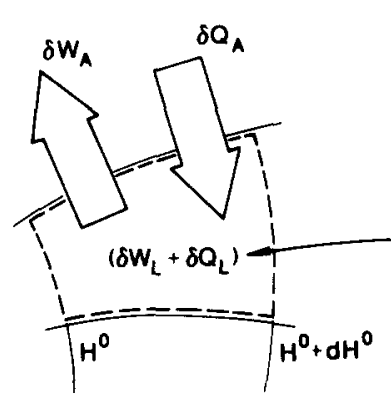

(a)

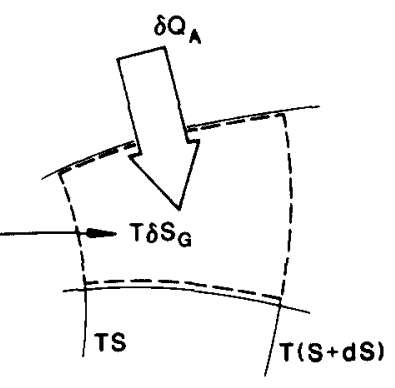

(b)

Fig. 2. (a) First Law in terms of available and lost work and heat. (b) Energy equivalent of Second Law balanced with entropy production. 


\section{LOCAL ENTROPY PRODUCTION}

Although the concept of lost heat continues to escape the attention of classical thermodynamicists, its counterpart in a nonequilibrium flow, identified as the (thermal part of) local entropy production, is well-known (see, for example, Arpaci and Larsen [6]). The following brief review on this production is for the convenience of the proceeding section.

The nonequilibrium aspects of entropy production require the explicit consideration of the momentum balance, as well as the conservation of mass and thermal energy. For the Stokesean fluid, this balance in terms of the usual nomenclature is

$\rho \frac{D v_{i}}{D t}=-\frac{\partial p}{\partial x_{i}}+\frac{\partial \tau_{i j}}{\partial x_{j}}+\rho f_{i}$.

The local entropy balance (the Second Law balanced by the local entropy production) is

$\rho \frac{D s}{D t}=-\frac{\partial}{\partial x_{i}}\left(\frac{q_{i}}{T}\right)+s^{\prime \prime \prime}$,

where $s^{m}$ denotes the local entropy production. Also, the conservation of total (thermomechanical) energy including the heat flux expressed in terms of the entropy flux,

$$
\begin{aligned}
\frac{\partial q_{i}}{\partial x_{i}} & \equiv \frac{\partial}{\partial x_{i}}\left[\left(\frac{q_{i}}{T}\right) T\right] \\
& =T \frac{\partial}{\partial x_{i}}\left(\frac{q_{i}}{T}\right)+\left(\frac{q_{i}}{T}\right) \frac{\partial T}{\partial x_{i}},
\end{aligned}
$$

is

$$
\begin{aligned}
\rho \frac{D}{D t}\left(u+\frac{1}{2} v_{i}^{2}\right)= & -\frac{\partial}{\partial x_{i}}\left[\left(\frac{q_{i}}{T}\right) T\right]-\frac{\partial}{\partial x_{i}}\left(p v_{i}\right) \\
& +\frac{\partial}{\partial x_{j}}\left(\tau_{i j} v_{i}\right)+\rho f_{i} v_{i}+u^{\prime \prime \prime},
\end{aligned}
$$

where $u^{m}$ denotes the local energy generation.

Now consider, the difference

Total energy $-($ Momentum $) v_{i}-($ Entropy $) T$, which, in terms of Eqs. (15), (i6), and (18) and the conservation of mass,

$\frac{D \rho}{D t}+\rho \frac{\partial v_{i}}{\partial x_{i}}=0$,

yields

$$
\begin{gathered}
\rho\left(\frac{D u}{D t}-T \frac{D s}{D t}+p \frac{D v}{D t}\right) \\
=-\left(\frac{q_{i}}{T}\right) \frac{\partial T}{\partial x_{i}}+\tau_{i j} s_{i j}+u^{m}-T s^{m},
\end{gathered}
$$

where $s_{i j}$ is the rate of deformation. For a reversible process, all forms of dissipation vanish, and

$$
\left(\frac{D u}{D t}-T \frac{D s}{D t}+p \frac{D v}{D t}\right)=0,
$$

which is the Gibbs Thermodynamic relation. For an irreversible process, Eq. (22) continues to hold provided the process can be assumed in local equilibrium. Then, the local entropy production is found to be

$s^{\prime \prime \prime}=\frac{1}{T}\left[-\left(\frac{q_{i}}{T}\right)\left(\frac{\partial T}{\partial x_{i}}\right)+\tau_{i j} s_{i j}+u^{\prime \prime}\right]$,

where the first term in brackets denotes the dissipation of thermal energy into entropy (lost heat), the second term denotes the dissipation of mechanical energy into heat (lost work), and the third term denotes the dissipation of any (except thermomechanical) energy into heat. In terms of the usual conductive constitution the local entropy production is found to be

$s^{m}=\frac{1}{T}\left[\frac{k}{T}\left(\frac{\partial T}{\partial x_{i}}\right)^{2}+\tau_{i j} s_{i j}+u^{m}\right]$.

The next section is devoted to an application of the foregoing concepts to flames.

\section{FLAME QUENCHING}

Consider a flat flame anchored to a porous-plug flameholder, suggested originally by Hirschfelder and coworkers [7-9] for experimental studies. Such flameholders were designed and utilized 
earlier by Botha and Spalding [10], Kaskan [11], and recently by Ferguson and Keck [12, 13]. The local entropy production in such a flame obtained from dimensional considerations on the thermal part of Eq. (23), is

$s^{m}-\frac{1}{T}\left(\frac{q^{K}}{T}\right)\left(\frac{T_{\mathrm{b}}-T_{\mathrm{u}}}{D}\right)$,

$D$ being the quench distance (the thickness of reaction zone is $d$, and $d<D$ ), and $T_{\mathrm{u}}$ and $T_{\mathrm{b}}$ unburned and burned gas temperatures, respectively (Fig. 3). Rearrange Eq. (25) in terms of the conduction law,

$q^{K} \sim k \frac{T_{\mathrm{b}}-T_{\mathrm{u}}}{D}$

as

$s^{m} \sim \frac{k}{T^{2}}\left(\frac{T_{b}-T_{u}}{D}\right)^{2}$

In view of the fact that most of the reaction occurs close to the highest temperature, use $T_{b}$ for the characteristic temperature in Eq. (27). Accordingly,

$s^{m} \sim\left(1-\frac{T_{\mathrm{u}}}{T_{\mathrm{b}}}\right)^{2} \frac{k}{D^{2}}$,

or, in terms of a characteristic length $l=\alpha / S_{\mathrm{u}}{ }^{0}, \alpha$ being the thermal diffusivity and $S_{\mathrm{u}}{ }^{0}$ the adiabatic laminar flame speed at the unburned gas temperature, assuming $T_{\mathrm{u}} / T_{\mathrm{b}}<1$ and introducing dimen-

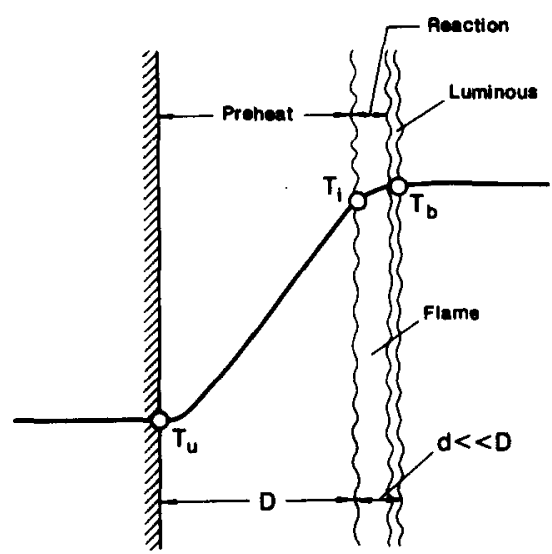

Fig. 3. Quenched laminar flame. sionless entropy production $\Pi_{s}$,

$\Pi_{s}=\frac{s^{m} l^{2}}{k} \sim\left(\mathrm{Pe}_{\mathrm{D}}\right)^{-2}$,

where

$\mathrm{Pe}_{\mathrm{D}}{ }^{0}=\frac{D}{l}=\frac{S_{\mathrm{u}}^{0} D}{\alpha}$

is the flame Peclet number. Accordingly,

$\Pi_{s}=f\left(\mathrm{Pe}_{\mathrm{D}}{ }^{0}\right)$,

where

$\mathrm{Pe}_{\mathrm{D}}{ }^{0}=f(D) \quad$ and $\quad D=f\left(\theta_{\mathrm{b}}\right)$,

and $\Pi_{s}$ depends on the flame temperature only through the Peclet number (or the dimensionless quench distance). The U-shaped nature of $D=$ $f\left(\theta_{b}\right)$ is well documented in the literature (see Ferguson and Keck [12, 13], Clarke and McIntosh [14], and McIntosh and Clarke [15] for the case excluding radiation, and Arpaci and Tabaczynski $[16,17]$ for the case including radiation; also, see Kooker [18] and Sohrab and Law [19] for the importance of radiation on quenching processes, and Lee and Tien [20] for the effect of condensed fuels on this process). References [12, 13, 16, 17] follow the usual practice and evaluate the minimum quench distance from the tangency condition,

$\frac{\partial}{\partial \theta_{b}}\left(\mathrm{Pe}_{\mathrm{D}}{ }^{0}\right)=0$,

which actually corresponds to an extremum in the entropy production, that is,

$\frac{\partial \Pi_{s}}{\partial \theta_{\mathrm{b}}} \sim-\frac{2}{\left(\mathrm{Pe}_{\mathrm{D}}{ }^{0}\right)^{3}} \frac{\partial}{\partial \theta_{\mathrm{b}}}\left(\mathrm{Pe}_{\mathrm{D}}{ }^{0}\right)=0$.

This result, in view of the fact that $\mathrm{Pe}_{\mathrm{D}}{ }^{0} \neq 0$, is equivalent to Eq. (32), and provides the physical justification for the tangency condition.

\section{DISTRIBUTION OF ENTROPY PRODUCTION}

So far we have discussed the foundations of entropy production in flame quenching following 
some dimensional considerations. Now we proceed to a qualitative distribution of this production by referring to a thermal model for steady plane flames on a porous plug. A number of simple models have been proposed, all describing the chemistry by a single-step global Arrhenius reaction, and differing especially in the way the heat losses are taken into account. Among these, Carrier et al. [21] use a step function heat sink in the preheat zone whereas Clarke and coworkers $[14,15,22]$ follow the model proposed earlier by Hirschfelder and coworkers [7-9]. The close agreement between these models, except for the interpretation of "cold boundary" (see, for example, Williams [23, p. 145]) and the model by Matkowsky and Olagunju [24] based on a modified step function, which yields results different in some important respects, are well-known. Also, there exist two models, proposed by Ferguson and Keck [12, 13], for interpretation of their experimental studies. The last two models are conveniently utilized here for a qualitative demonstration of the entropy production in flames. Following Ref. [12], we have the first order model

$$
\begin{aligned}
\mathrm{Pe}_{\mathrm{D}}{ }^{0} & =\frac{\rho_{\mathrm{u}} S_{\mathrm{u}}{ }^{0} c_{\mathrm{p}} D}{k} \\
& =\left(\frac{\theta_{\mathrm{b}}-\theta_{\mathrm{u}}}{1+\theta_{\mathrm{b}}}\right) \exp \left[\frac{E}{2 R T_{\mathrm{b}}{ }^{0}}\left(\frac{1}{\theta_{\mathrm{b}}}-1\right)\right],
\end{aligned}
$$

where $\theta_{\mathrm{u}}=T_{\mathrm{u}} / T_{\mathrm{b}}{ }^{0}$ and $\theta_{\mathrm{b}}=T_{\mathrm{b}} / T_{\mathrm{b}}{ }^{0}$ are dimensionless temperatures, $T_{\mathrm{b}}{ }^{0}$ being the adiabatic flame tempdrature, $E$ the activation energy, and $R$ the universal gas constant. Also, following Ref. [13] we have the second order model,

$$
\begin{aligned}
\mathrm{Pe}_{\mathrm{D}}{ }^{0}= & \rho_{\mathrm{u}} S_{\mathrm{u}}{ }^{0} c_{\mathrm{p}} \int_{0}^{D} \frac{d x}{k}=\left[\ln \left(\frac{1-\theta_{\mathrm{u}}}{1-\theta_{\mathrm{b}}}\right)\right] \\
& \times \exp \left[\frac{E}{2 R T_{\mathrm{b}}{ }^{0}}\left(\frac{1}{\theta_{\mathrm{b}}}-1\right)\right] .
\end{aligned}
$$

Now, employing Eqs. (34) and (35), we get the distribution of entropy production from Eq. (29) and plot the results in Fig. 4. On the same figure, also shown is the variation of Peclet number. The

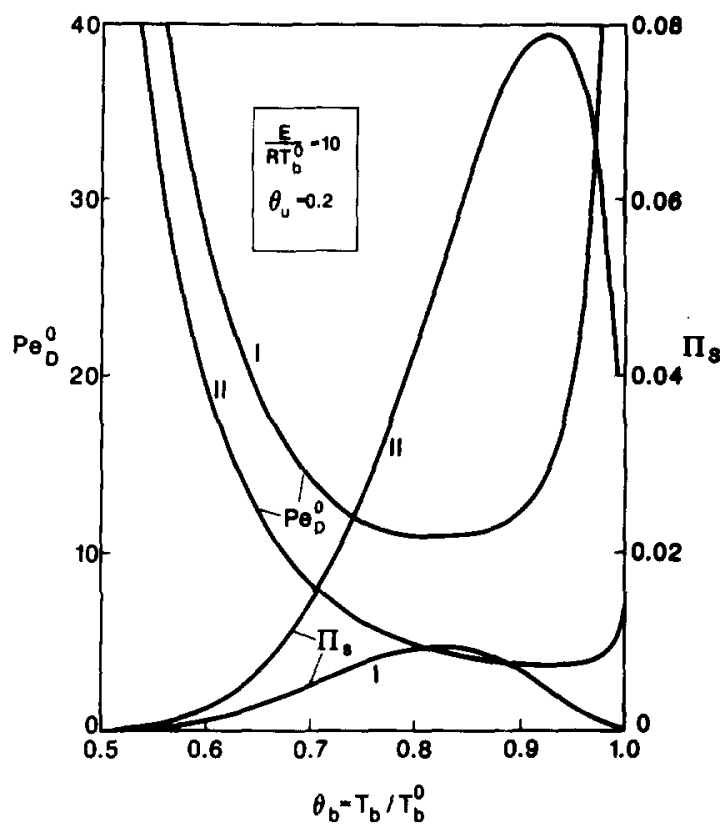

Fig. 4. First and second order $\mathrm{Pe}_{\mathrm{D}}{ }^{0}$ and $\theta_{\mathrm{b}}=T_{\mathrm{b}} / T_{\mathrm{b}}{ }^{0}$.

Peclet and entropy production curves labeled by I and II correspond to the first and second order models evaluated, respectively, from Eqs. (34) and (35). The U-shape of the $\mathrm{Pe}_{\mathrm{D}}{ }^{0}-\theta_{\mathrm{b}}$ relation and the inverse quadratic dependence between $\Pi_{s}$ and $\mathrm{Pe}_{\mathrm{D}}{ }^{0}$ [recall Eq. (29)] readily explain the maximum as well as the relatively squeezed shape of the $\Pi_{s}-\theta_{\mathrm{b}}$ relation.

For the spatial distribution of entropy production between the burner and flame, consider the temperature distribution from Ref. [13],

$\frac{T-T_{\mathrm{u}}}{T_{\mathrm{b}}{ }^{0}-T_{\mathrm{u}}}=\left(\frac{T_{\mathrm{b}}-T_{\mathrm{u}}}{T_{\mathrm{b}}{ }^{0}-T_{\mathrm{u}}}\right)\left(\frac{e^{\xi}-1}{e^{\mathrm{Pe} \mathrm{D}}-1}\right)$,

where

$\xi=\rho_{\mathrm{u}} S_{\mathrm{u}} c_{\mathrm{p}} \int_{0}^{x} \frac{d x^{\prime}}{k}$.

Rearrange Eq. (36) in terms of

$\theta=\frac{T}{T_{\mathrm{b}}{ }^{0}} \quad$ and $\quad \mathrm{Pe}_{\mathrm{D}}=\ln \left(\frac{1-\theta_{\mathrm{u}}}{1+\theta_{\mathrm{b}}}\right)$

to obtain

$\theta(\xi)=\theta_{\mathrm{u}}+\theta_{\mathrm{b}}-1+\left(1-\theta_{\mathrm{b}}\right) e^{\xi}$ 
and

$\frac{d \theta}{d \xi}=\left(1-\theta_{\mathrm{b}}\right) e^{\xi}$

Now, for the thermal part of entropy production, Eq. (24) gives

$\Pi_{s}=\frac{s^{\prime \prime \prime} l^{2}}{k}=\frac{l^{2}}{T^{2}}\left(\frac{d T}{d x}\right)^{2}$,

which may be rearranged as

$\Pi_{s}=\frac{1}{\theta^{2}}\left[\frac{d \theta}{d(x / l)}\right]^{2}$.

In terms of $l=\alpha / S_{u}{ }^{0}$ and for a constant $k$,

$\frac{x}{l}=\frac{\rho_{u} c_{p} S_{u}{ }^{0} x}{k}=\frac{S_{u} x / \alpha}{S_{u} / S_{u}{ }^{0}}$,

or

$\frac{x}{l}=\frac{\xi}{S_{\mathrm{u}} / S_{\mathrm{u}}{ }^{0}}$.

Now, in terms of $\xi$, Eq. (42) becomes

$\Pi_{s}=\frac{1}{\theta^{2}}\left(\frac{d \theta}{d \xi}\right)^{2}\left(\frac{S_{\mathrm{u}}}{S_{\mathrm{u}}{ }^{0}}\right)^{2}$,

which, with the experimental correlation

$\frac{S_{\mathrm{u}}}{S_{\mathrm{u}}{ }^{0}}=\exp \left[-\frac{E}{2 R T_{\mathrm{b}}{ }^{0}}\left(\frac{1}{\theta_{\mathrm{b}}}-1\right)\right]$

of Kaskan [11], yields

$\Pi_{s}=\frac{1}{\theta^{2}}\left(\frac{d \theta}{d \xi}\right)^{2} \exp \left[-\frac{E}{R T_{\mathrm{b}}{ }^{0}}\left(\frac{1}{\theta_{\mathrm{b}}}-1\right)\right]$.

Finally, referring to Eqs. (39) and (40) for $\theta$ and $d \theta / d \xi$, Eq. (46) may be rearranged as

$$
\begin{aligned}
\Pi_{s}= & {\left[\frac{\left(1-\theta_{\mathrm{b}}\right) e^{\xi}}{\theta_{\mathrm{u}}+\theta_{\mathrm{b}}-1+\left(1-\theta_{\mathrm{b}}\right) e^{\xi}}\right]^{2} } \\
& \times \exp \left[-\frac{E}{R T_{\mathrm{b}}^{0}}\left(\frac{1}{\theta_{\mathrm{b}}}-1\right)\right] .
\end{aligned}
$$

Note that for

$\theta_{\mathrm{u}}+\theta_{\mathrm{b}}=1$

Eq. (47) reduces to

$\Pi_{s}=\exp \left[-\frac{E}{R T_{\mathrm{b}}{ }^{0}}\left(\frac{1}{\theta_{\mathrm{b}}}-1\right)\right]$,

which, for a fixed $E / R T_{\mathrm{b}}{ }^{0}$ and $\theta_{\mathrm{b}}$, becomes constant. Figure 5 shows $\Pi_{s}$ versus $\xi / \mathrm{Pe}_{\mathrm{D}}$ for $\theta_{\mathrm{u}}=$ 0.2 and $E / R T_{\mathrm{b}}{ }^{0}=10$. The entropy production between the flame and burner appears to be almost uniform. Since the quench distance is rather small, say $0.5-1 \mathrm{~mm}$ (see Ref. [13]), this result is not surprising. For $\theta_{\mathrm{b}}=1-\theta_{\mathrm{u}}$, this production becomes exactly uniform. However, for $\theta_{\mathrm{b}}>1-$ $\theta_{\mathrm{u}}$, the behavior of production drastically changes as demonstrated in Fig. 5 with $\theta_{\mathrm{b}}=0.96$. The uniformity of, as well as the drastic change in, entropy production does not accept a ready interpretation. It may be more a property of the model rather than the reality. The quantitative difference between the model and experimental results (see Fig. 6 of Ref. [13]) for $\theta_{\mathrm{b}}>1-\theta_{\mathrm{u}}$ adds some credence to this statement.

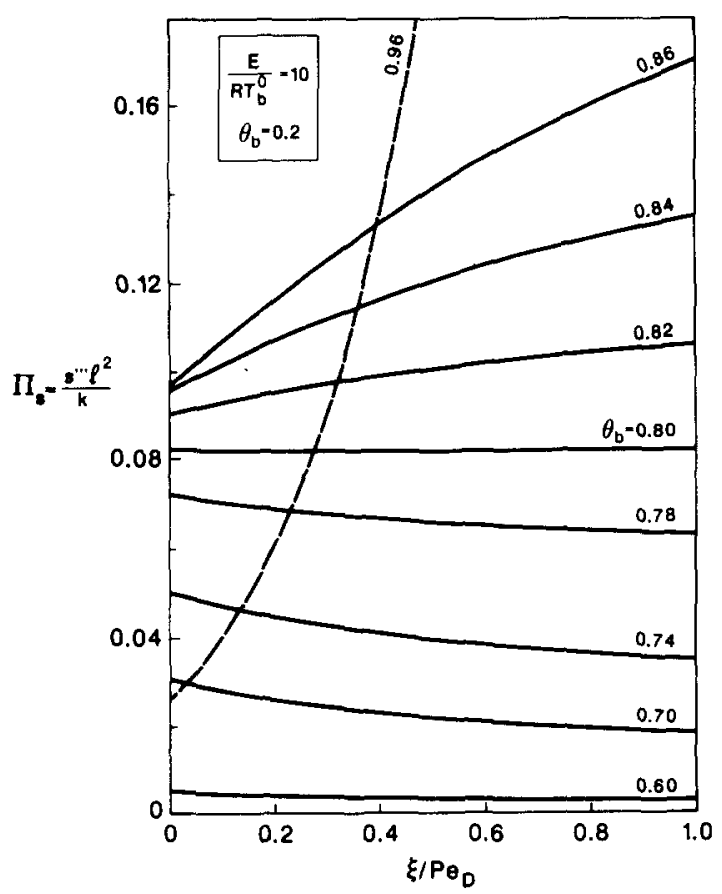

Fig. 5. Spatial distribution of entropy production. 
Among the flame models existing in the literature, the foregoing Ferguson and Keck models were employed because of their simplicity. A third and somewhat more involved model, based on studies of Clarke and coworkers [14, 15, 22], and resting on the flame speed

$$
\frac{S_{\mathrm{u}}}{S_{\mathrm{u}}{ }^{0}}=\left(\frac{T_{\mathrm{b}}}{T_{\mathrm{b}}{ }^{0}}\right)^{2} \exp \left[-\frac{E}{2 R T_{\mathrm{b}}{ }^{0}}\left(\frac{1}{\theta_{\mathrm{b}}}-1\right)\right],
$$

readily yields

$$
\begin{aligned}
\mathrm{Pe}_{\mathrm{D}}{ }^{0}= & \frac{1}{\theta_{\mathrm{b}}{ }^{2}} \ln \left(\frac{1-\theta_{\mathrm{u}}}{1-\theta_{\mathrm{b}}}\right) \\
& \times \exp \left[\frac{E}{2 R T_{\mathrm{b}}^{0}}\left(\frac{1}{\theta_{\mathrm{b}}}-1\right)\right],
\end{aligned}
$$

or

$$
\left(\mathrm{Pe}_{\mathrm{D}}{ }^{0}\right)_{\text {III }}=\theta_{\mathrm{b}}^{-2}\left(\mathrm{Pe}_{\mathrm{D}}{ }^{0}\right)_{\mathrm{II}}
$$

and

$$
\left(\Pi_{s}\right)_{\mathrm{III}}=\theta_{\mathrm{b}}^{4}\left(\Pi_{s}\right)_{\mathrm{II}} \text {, }
$$

where subscripts II and III, respectively, refer to the second order Ferguson and Keck model and the Clarke model. A numerical comparison between these models shows that, in spite of its relative complexity, the Clarke model relative to the Ferguson and Keck models appears to provide a limited improvement in approximating real flames.

The range of $\theta_{\mathrm{u}}$ is $0.12-0.25$ and the range of $E / R T_{\mathrm{b}}{ }^{0}$ for ordinary hydrocarbon fuels (say, methane, propane, and octane) reacting with air is

$E / R T_{\mathrm{b}}{ }^{0}=5-15$.

Figure 6 shows the variation of $\mathrm{Pe}_{\mathrm{D}}{ }^{0}$ and $\Pi_{s}$ based on the second order Ferguson and Keck model. Here, $E / R T_{\mathrm{b}}{ }^{0}=10$ qualitatively represents (for the stoichiometric mixture with air) a lower bound for propane and an upper bound for (n-octane), and $E / R T_{\mathrm{b}}{ }^{\circ}=15$ an upper bound for methane. Actually, there remains a considerable disagreement in the literature on the activation energy of methane (see, for example, the tables in Kanury

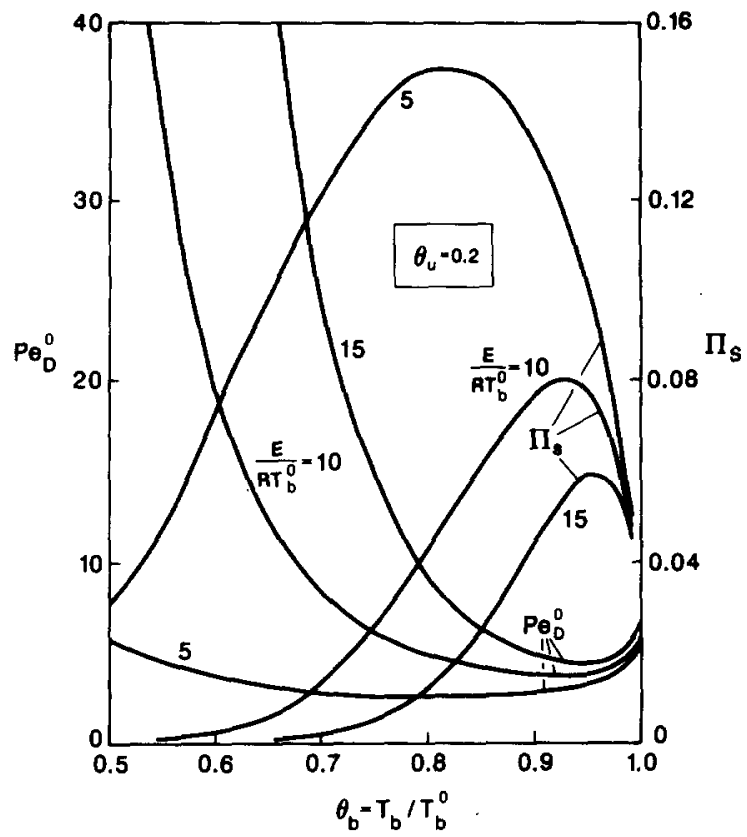

Fig. 6. Effect of activation energy on $\mathrm{Pe}_{\mathrm{D}}{ }^{0}$ and $\Pi_{s}$.

[25, p. 109], Mullins [26, pp. 201-202], and Kaskan [11]). This disagreement is a result of the difficulties associated with $\mathrm{CH}_{4}$ oxidation (Westbrook and Dryer [27] and Glassman [28, p. 81]). Here, we utilized, somewhat arbitrarily, the values suggested by Kaskan [11].

\section{CONCLUSIONS}

The concept of lost heat is originated as opposed to that of lost work. It is shown that all forms of energy are dissipated into heat and describe the nonthermal part of entropy production while the heat energy is dissipated into entropy and describes the thermal part of this production. A dimensionless number for entropy production is introduced. This number is evaluated in terms of an illustrative case which involves the entropy production in the luminous zone of a quenched flame. The production is found to be inversely proportional to the Peclet number. The tangency condition, usually considered in the literature to determine the minimum quench distance, is related to an extremum of entropy production. The distribution of entropy production between the flame and burner appears to remain constant for $\theta$ 
$<1-\theta_{u}$ This result is not surprising in view of the magnitude of the quench distance. The rapid and unusual change in distribution of entropy production for $\theta_{\mathrm{b}} \geq 1-\theta_{\mathrm{u}}$ is quite surprising and does not appear to be readily justifiable. This may well be a result of the nature of the models which for $\theta_{\mathrm{b}}>1-\theta_{\mathrm{u}}$ begin to deviate from experimental results as shown in Ref. [13].

Future research on real flame entropy should first be related to existing models of the prediction of flame structure rather than to one-dimensional models (such as those employed in this study) which are based on the assumption of negligible flame thickness. Examples of models incorporating flame structure are the studies by Westbrook and Dryer [27], and Warnatz, Miller, Kee, and coworkers (see Ref. [29]).

\section{REFERENCES}

1. Bejan, A., Entropy Generation through Heat and Fluid Flow, Wiley, New York, 1982.

2. Bejan, A., Adv. Heat Transfer 15:1-58 (1982).

3. Arpaci, V. S., $A I A A J$. 24:1859-1860 (1986).

4. Arpaci, V. S., Int. J. Heat Mass Transfer 30:21152123 (1987).

5. Arpaci, V. S., and Selamet, A., Proc. Eighth Int. Heat Transfer Conf. 2:729-734 (1986).

6. Arpaci, V. S., and Larsen, P. S., Convection Heat Transfer, Prentice-Hall, New Jersey, 1984, pp. 40-46.

7. Hirschfelder, J. O., and Curtiss, C. F., Journal of Chemical Physics 17:1076-1081 (1949).

8. Hirschfelder, J. O., and Curtiss, C. F., Third Symposium (International) on Combustion, Williams and Wilkins, Baltimore, 1949, pp. 121-127.

9. Hirschfelder, J. O., Curtiss, C. F., and Campbell, D. E., Fourth Symposium (International) on Combustion, Williams and Wilkins, Baltimore, 1953, pp. 190-211.

10. Botha, J. P., and Spalding, D. B., Proc. Roy. Soc. Lond. A225:71-96 (1954).
11. Kaskan, W. E., Sixth Symposium (International) on Combustion, Reinhold, New York, 1957, pp. 134-143.

12. Ferguson, C. R., and Keck, J. C., Combust. Flame 28:197-205 (1977).

13. Ferguson, C. R., and Keck, J. C., Combust. Flame 34:85-98 (1979).

14. Clarke, J. F., and McIntosh, A. C., Proc. Roy. Soc. Lond. A372:367-392 (1980).

15. McIntosh, A. C., and Clarke, J. F., Combustion Science and Technology 37:201-219 (1984).

16. Arpaci, V. S., and Tabaczynski, R. J., Combust. Flame 46:315-322 (1982).

17. Arpaci, V. S., and Tabaczynski, R. J., Combust. Flame 57:169-178 (1984).

18. Kooker, D. E., Combust. Flame 49:141-149 (1983).

19. Sohrab, S. H., and Law, C. K., Int. J. Heat Mass Transfer 27:291-300 (1984).

20. Lee, K. Y., and Tien, C. L., Combustion Science and Technology 43:167-182 (1985).

21. Carrier, G. F., Fendell, F. E., and Bush, W. B., Combustion Science and Technology 18:33-46 (1978).

22. Clarke, J. F., Combust. Flame 50:125-138 (1983).

23. Williams, F. A., Combustion Theory, 2nd Ed., Benjamin/Cummnings, Menlo Park, California, 1985.

24. Matkowsky, B. J., and Olagunju, D. O., SIAM J. Appl. Math. 40:551-562 (1981).

25. Kanury, A. M., Introduction to Combustion Phenomena, Gordon and Breach Science, New York, 1975.

26. Mullins, B. P., in Explosions, Detonations, Flammability and Ignition (S. S. Penner and B. P. Mullins, Eds., AGARDograph No. 31, Pergamon, New York, 1959.

27. Westbrook, C. K., and Dryer, F. L., Prog. Energy Combust. Sci. 10:1-57 (1984).

28. Glassman, I., Combustion, 2nd. edn., Academic Press, Orlando, Florida, 1987.

29. Peters, N., and Warnatz, J. (Eds.), Numerical Methods in Laminar Flame Propagation, Vieweg \& Sohn, Braunschweig/Wiesbaden, Germany, 1982.

Received 29 June 1987; revised 14 December 1987 